\title{
Topological rigidity of semigroups of affine maps
}

\author{
ALEX CLARK*† and ROBBERT FOKKINK \\ $\dagger$ Faculty of Mathematics, University of North Texas, Denton, Texas, USA \\ $\$$ Faculty of Electrical Engineering, Mathematics and Information Technology, \\ Delft University, PO Box 5031, 2600 GA Delft, The Netherlands
}

(Received 18 April 2006; in final form 22 September 2006)

\begin{abstract}
We study the topological rigidity of affine semigroups of algebraic actions. In the first part of the paper we given an algebraic condition for rigidity that unifies previous rigidity results and we settle an old question of Walters. In the second part we study a generalized notion of the rigidity of hyperbolic actions.
\end{abstract}

\section{Introduction}

For our purposes, an algebraic dynamical system $(X, S)$ is an action of a discrete commutative semigroup with unity $S$ (a monoid) acting by affine transformations of a compact connected abelian metrisable group $X$. The group operation of $X$ is denoted additively and the semigroup operation of $S$ is denoted multiplicatively. Given algebraic dynamical systems $(X, S)$ and $(Y, S)$, a continuous map $f: X \rightarrow Y$ is equivariant if $f(s \cdot x)=s \cdot f(x)$ for all $s \in S$. An algebraic dynamical system $(Y, S)$ is topologically rigid if all equivariant maps into $Y$ are affine; i.e. if $f: X \rightarrow Y$ is equivariant for any $X$, then $f(x)=y+A(x)$ for some $y \in Y$ and a homomorphism $A$ between $X$ and $Y$. Usually the notion is restricted to a subclass of all $S$-algebraic dynamical systems. For instance, if $(Y, S)$ is ergodic, then all $(X, S)$ considered are assumed to be ergodic as well.

Let $\mathbb{Z}[S]$ be the semigroup ring that consists of all finite formal sums of elements of $S$ with natural multiplication. If $S$ acts by endomorphisms, then $X$ has the structure of a $\mathbb{Z}[S]$-module. An algebraic dynamical system does not necessarily have this structure since $S$ acts by affine transformations, but this can be remedied. If $T$ is affine, then $T(x)=a+A(x)$ for a uniquely determined homomorphism $A$, and we say that $A$ is the linear part of $T$. If $S$ acts on $X$ by affine transformations, then we can give $X$ the structure of a $\mathbb{Z}[S]$-module by replacing the affine transformations $s \mapsto s \cdot x$ by their linear parts. By Pontryagin duality this $\mathbb{Z}[S]$-module structure carries over to the character group $\hat{X}$.

The linear space $C(X, \mathbb{R})$ of continuous maps inherits the structure of a $\mathbb{Z}[S]$-module from the action of $S$ defined by $s \cdot f(x)=f(s \cdot x)$. Define $C_{0}(X, \mathbb{R})$ to

*Corresponding author. Email: alexc@unt.edu 
be the submodule of all functions for which $\int f \mathrm{~d} \mu=0$ for the normalized Haar measure $\mu$. In the first part of the paper we show that the rigidity of $Y$ depends on the $\mathbb{Z}[S]$-module structure of $C_{0}(X, \mathbb{R})$, where $X$ ranges over the subclass of $S$-dynamical systems. More specifically, we obtain the following algebraic characterization of rigidity that unifies previous results of [1-4].

Theorem 1.1: Let $\mu$ be the Haar measure on $X$ and let $C_{0}(X, \mathbb{R}) \subset C(X, \mathbb{R})$ be the submodule of all functions for which $\int f \mathrm{~d} \mu=0$. All equivariant maps from $X$ to $Y$ are affine if and only if the only $\mathbb{Z}[S]$-module homomorphism between $\hat{Y}$ and $C_{0}(X, \mathbb{R})$ is the trivial homomorphism.

We are indebted to [3], which implicitly contains this theorem for the case that $S$ is equal to $\mathbb{Z}^{d}$ and acts by automorphisms. The techniques we use can be considered as an extension of the techniques used in [2].

It is not surprising that many algebraic dynamical systems are rigid: if $S$ acts topologically transitively on $X$, which it does if the action is ergodic (with respect to the Haar measure), then an equivariant $f$ is determined by a single value. So it is natural to study rigidity under a condition less restrictive than equivariance. In the second part of this paper we introduce such a condition for hyperbolic dynamical systems. In this case $S$ is equal to $\mathbb{Z}^{d}$ and it acts on $X$ by automorphisms. With $W_{X}^{S}=\left\{x \in X: \lim _{n \rightarrow \infty} n \cdot x=0\right\}$ and $W_{X}^{u}=\left\{x \in X: \lim _{n \rightarrow-\infty} n \cdot x=0\right\}$ denoting the stable and unstable groups of $X$, we say a map $f: X \rightarrow Y$ between hyperbolic algebraic systems respects the hyperbolic structure if $f$ is continuous, $f(0)=0$ and $f\left(W_{X}^{s}\right) \subset W_{Y}^{s}$ and $f\left(W_{X}^{u}\right) \subset W_{Y}^{u}$.

Theorem 1.2: $\quad$ Suppose that $X$ is a hyperbolic algebraic system such that the path components of the stable and the unstable groups are dense. Then every continuous map $f: X \rightarrow Y$ that respects the hyperbolic structure is a homomorphism.

\section{Topological rigidity of algebraic dynamical systems}

Let $\mathbb{T}$ denote the circle group, so the character group $\hat{X}$ is equal to $\operatorname{Hom}(X, \mathbb{T})$ with the compact-open topology. Lin [5] observed that $\operatorname{Hom}(X, \mathbb{R})$ is very similar to the tangent space $T_{p} M$ of a manifold $M$.

Definition 2.1: The tangent space $L(X)$ of a compact connected abelian group $X$ is the group of functionals $\operatorname{Hom}(\hat{X}, \mathbb{R})$ with the compact-open topology. The exponential map from $\mathbb{R}$ to $\mathbb{T}$ induces a homomorphism exp: $L(X) \rightarrow X$ onto the path-component of the identity $0 \in X$.

Let $A$ be an endomorphism on $X$ and let $\hat{A}$ be the dual action on $\hat{X}$. Then $\varphi \mapsto \varphi \circ \hat{A}$ is an operator on $L(X)$ satisfying $\exp \circ L(A)=A \circ \exp$, so $L(A)$ is a lift of $A$ to $L(X)$. It follows that the $\mathbb{Z}[S]$-module structure on $X$ carries over to the tangent space.

Theorem 2.2 [5]: The homomorphism exp: $L(X) \rightarrow X$ has the homotopy lifting property.

In other words, a null-homotopic map $f: Y \rightarrow X$ can be factored through a map $F: Y \rightarrow L(X)$ such that $f=\exp \circ F$. If $Y$ is a topological group and $f$ is a homomorphism, then by connectedness $F$ is a homomorphism and $F(Y)$ is a 
compact subgroup of a linear space. Hence $F(Y)$ is trivial and we find that a null-homotopic homomorphism from $Y$ to $X$ is trivial. It follows that any continuous map from $Y$ to $X$ is homotopic to at most one homomorphism. Gorin [6] and Scheffer [7] proved that in the base-point preserving category, every homotopy class indeed contains a homomorphism.

Theorem 2.3 (Gorin and Scheffer): Let $X, Y$ be compact and connected abelian groups. Then each continuous $f: X \rightarrow Y$ for which $f(0)=0$ is homotopic to a unique homomorphism.

This Gorin-Scheffer theorem generalizes the theorem of Van Kampen, which is used in $[3,4]$.

A vector valued function $f: X \rightarrow L(Y)$ can be integrated with respect to the Haar measure. We denote this integral by $\int f \mathrm{~d} \mu$. It is an element of $L(Y)$.

Proposition 2.4: $\quad$ Let $A$ be an endomorphism on $Y$ and let $L(A)$ be its lifted operator on $L(Y)$. For any continuous $f: X \rightarrow L(Y)$ we have that $L(A)\left(\int f \mathrm{~d} \mu\right)=\int L(A) \circ f \mathrm{~d} \mu$.

Proof: By our assumptions, $f(x)$ is a functional on $\hat{Y}$ for each $x \in X$. The integral $\int f \mathrm{~d} \mu$ is the functional that maps the character $\chi \in \hat{X}$ to $\int f(x)(\chi) \mathrm{d} \mu$. The action of the operator $L(A)$ on $L(Y)$ is induced by the action $\chi \mapsto \rightarrow \chi \circ A$ on $\hat{Y}$. Hence $L(A)\left(\int f \mathrm{~d} \mu\right)$ maps $\chi$ to $\int f(x)(\chi \circ A) \mathrm{d} \mu$, as does $\int L(A) \circ f \mathrm{~d} \mu$.

If $S$ is equal to $\mathbb{N}$ then the action on $X$ is induced by a single affine transformation $T$. We denote the algebraic dynamical system by $(X, T)$ in this case.

Theorem 2.5: Let $\left(X, T_{1}\right)$ and $\left(Y, T_{2}\right)$ be equivariant algebraic dynamical systems. Let $A_{2}$ be the linear part of $T_{2}$. Then every equivariant map from $X$ to $Y$ is affine if and only if each $f: X \rightarrow L(Y)$ such that $f \circ T_{1}=L\left(A_{2}\right) \circ f$ is constant.

Proof: We say that a map $f: X \rightarrow L(Y)$ is equivariant if $f \circ T_{1}=L\left(A_{2}\right) \circ f$. It is obvious that the composition of such an equivariant map $f$ and exp induces an equivariant map between $\left(X, T_{1}\right)$ and $\left(Y, A_{2}\right)$. If $h: X \rightarrow Y$ is an equivariance, then so is $x \mapsto h(x)+\exp \circ f(x)$. If $f$ is not constant, then neither is exp $\circ f$. Hence if every equivariant map between $X$ and $Y$ is affine, then every equivariant map between $X$ and $L(Y)$ is constant.

Assume that all equivariant maps between $X$ and $L(Y)$ are constant. We have to show that an equivariant map $g: X \rightarrow Y$ is affine. By the Gorin-Scheffer theorem (Theorem 2.3) there exists a homomorphism $E: X \rightarrow Y$ that is homotopic to $g-g(0)$. Since $g \circ T_{1}-T_{2} \circ g$ is constant, $E \circ T_{1}-T_{2} \circ E$ is homotopic to a constant map. But $E \circ T_{1}-T_{2} \circ E$ is an affine map, so it must be a constant map $x \mapsto c$ for some $c \in Y$.

Define $h(x)=g(x)-E(x)-g(0)$. Note that $h$ is a null-homotopic map. Now compute

$$
\begin{aligned}
h\left(T_{1}(x)\right) & =g\left(T_{1}(x)\right)-E\left(T_{1}(x)\right)-g(0) \\
& =T_{2}(g(x))-T_{2}(E(x))-c-g(0) \\
& =A_{2}(g(x)-E(x))-c-g(0) \\
& =A_{2}(g(x)-E(x)-g(0))-c+A_{2}(g(0))-g(0) \\
& =A_{2}(h(x))-c+A(g(0))-g(0) .
\end{aligned}
$$


So $h \circ T_{1}-A_{2} \circ h$ is constant. Now $h$ is null-homotopic, so by Lin's theorem

(Theorem 2.2) $h=\exp \circ \tilde{h}$ for some $\tilde{h}: X \rightarrow L(Y)$ and by the equation above $\tilde{h} T_{1}-L\left(A_{2}\right) \tilde{h}$ is constant, say equal to $\tilde{c}$. By the invariance of the Haar measure $\int \tilde{h} \mathrm{~d} \mu=\int \tilde{h}\left(T_{1}(x)\right) \mathrm{d} \mu$. Since $\tilde{h} \circ T_{1}-L\left(A_{2}\right) \circ \tilde{h}$ is equal to $\tilde{c}$, by the proposition above we find that $\int \tilde{h} \mathrm{~d} \mu-L\left(A_{2}\right)\left(\int \tilde{h} \mathrm{~d} \mu\right)=\tilde{c}$. It follows that the map $f(x)=\tilde{h}(x)-\int \tilde{h} \mathrm{~d} \mu$ is equivariant from $X$ to $L(Y)$, hence it is constant. This implies that $g-E$ is constant, so $g$ is affine.

Corollary 2.6: Let $X$ and $Y$ be algebraic dynamical systems. Then every equivariant map from $X$ to $Y$ is affine if and only if each $S$-equivariant map between $X$ and $L(Y)$ is constant.

Proof: For any $s \in S$, apply Theorem 2.5 to the affine transformations $x \mapsto s \cdot x$ on $X$ and $y \mapsto s \cdot y$ on $Y$.

Walters [4] proved that an affine ergodic transformation $T: X \rightarrow X$ is rigid if $X$ is finite-dimensional, in the sense that every $T$-equivariant map $f: X \rightarrow X$ is affine. The following example provides a negative answer to a question of Walters (see p. 100 of [4]) that had apparently remained unsettled: if $(Y, T)$ is rigid and if $T$ is ergodic, is it true that $\hat{Y}$ is torsion?

Example 2.7: Let $\sigma$ be the shift of $\mathbb{T}^{\mathbb{Z}}$ defined by $\sigma\left(x_{n}\right)=\left(x_{n+1}\right)$. Then $\tau=2 \sigma$ rigid in the sense that any $\tau$-equivariant $\operatorname{map} f: \mathbb{T}^{\mathbb{Z}} \rightarrow \mathbb{T}^{\mathbb{Z}}$ is affine.

Proof: The tangent space of $\mathbb{T}^{\mathbb{Z}}$ is equal to $\mathbb{R}^{\mathbb{Z}}$ and the induced operator $L(\tau)$ is equal to $\left(r_{n}\right) \mapsto\left(2 r_{n+1}\right)$. In particular, $L(\tau)$ is bijective. If $f: \mathbb{T}^{\mathbb{Z}} \rightarrow \mathbb{R}^{\mathbb{Z}}$ is equivariant, then $f(x)=f(0)$ for any $x$ such that $\tau^{n}(x)=0$ for some $n \in \mathbb{N}$. It is not hard to check that the $x$ 's with this property form a dense subgroup of $\mathbb{T}^{\mathbb{Z}}$. So $f$ is constant and by Theorem $2.5 \tau$ is rigid.

Theorem 2.8: All equivariant maps from $X$ to $Y$ are affine if and only if the only $\mathbb{Z}[S]$-module homomorphism between $\hat{Y}$ and $C_{0}(X, \mathbb{R})$ is the trivial homomorphism.

Proof: First observe that $C_{0}(X, \mathbb{R})$ is a quotient of $C(X, \mathbb{R})$ under the projection $f \mapsto f-\int f \mathrm{~d} \mu$, so an equivariant map between $\hat{Y}$ and $C(X, \mathbb{R})$ projects onto an equivariant map between $\hat{Y}$ and $C_{0}(X, \mathbb{R})$. This projection is trivial if and only if the image of $\hat{Y}$ in $C(X, \mathbb{R})$ consists of constant maps.

It suffices to prove the theorem for systems with a single affine transformation $\left(X, T_{1}\right)$ and $\left(Y, T_{2}\right)$. The general case follows just as Corollary 2.6 follows from Theorem 2.5. Since $L(Y)$ is equal to $\operatorname{Hom}(\hat{Y}, \mathbb{R})$ an equivariant map $f: X \rightarrow L(Y)$ induces a map $\tilde{f}: X \times \hat{Y} \rightarrow \mathbb{R}$, defined by $(x, \chi) \mapsto f(x)(\chi)$. It is continuous in the first coordinate, additive in the second coordinate, and has the property that $\tilde{f}\left(x, \chi \circ A_{2}\right)=\tilde{f}\left(T_{1}(x), \chi\right)$.

For each fixed character $\chi$ the real-valued map $x \mapsto \tilde{f}(x, \chi)$ is continuous. Denote this map by $f_{\chi}$. Then $\chi \mapsto f_{\chi}$ is an equivariance between $\hat{Y}$ and $C(X, \mathbb{R})$. Conversely, any such equivariance induces an equivariant $f: X \rightarrow L(Y)$ defined by $f(x)(\chi)=f_{\chi}(x)$. The maps $f_{\chi}$ are constant for all $\chi$ if and only if $f$ is constant. By Theorem 2.5 equivariant maps between $X$ and $Y$ are affine if and only if the image of equivariant maps between $\hat{Y}$ and $C(X, \mathbb{R})$ are in the 
subset of constant maps. The result now follows by the projection of $C(X, \mathbb{R})$ to $C_{0}(X, \mathbb{R})$.

Now we show how the rigidity results of Walters [4] and Bhattacharya and Ward [3] relate to Theorem 2.8.

Theorem 2.9 (Bhattacharya and Ward): Suppose $S$ is $\mathbb{Z}^{d}$, and consider the subcategory of $S$-actions that are mixing. If $\hat{Y}$ is a Noetherian module, then $Y$ is rigid if and only if $\hat{Y}$ is a torsion module.

Proof: The proof depends on some non-trivial observations on the algebraic structure of $C_{0}(X, \mathbb{R})$ and $\hat{Y}$. In Lemma 4.2 of [3] it is shown that $C_{0}(X, \mathbb{R})$ is torsion-free if $X$ is mixing. So if $\hat{Y}$ is torsion then the trivial homomorphism is the only homomorphism between $\hat{Y}$ and $C_{0}(X, \mathbb{R})$.

If $\hat{Y}$ is not a torsion module, then $\hat{Y} / \operatorname{Tor}(\hat{Y})$ is a non-trivial torsion-free module. By Lemma 2.2 of [3], it embeds as a submodule of a free module, since $\hat{Y}$ is Noetherian. Hence $\hat{Y} / \operatorname{Tor}(\hat{Y})$ maps non-trivially to $C_{0}(X, \mathbb{R})$ for any non-trivial $X$.

Walters also considers the case that $\hat{Y}$ is a torsion module and that $S$ is equal to $\mathbb{N}$. His rigidity result is easiest to state by extension of the base, replacing $\hat{Y}$ by $\mathbb{R} \otimes_{\mathbb{Z}} \hat{Y}$. Since the action on $Y$ is generated by a single affine transformation $T, \mathbb{R} \otimes_{\mathbb{Z}} \hat{Y}$ is a vector space on which $A$, the linear part of $T$, acts.

Recall that $f: X \rightarrow \mathbb{C}$ is an eigenfunction of $(X, T)$ if $f(T x)=\lambda f(x)$ for some $\lambda \in \mathbb{C}$.

Theorem 2.10 (Walters [4]): If $\hat{Y}$ is torsion, then each equivariant map from $\left(X, T_{1}\right)$ to $\left(Y, T_{2}\right)$ is affine if there exists no eigenfunction $f \in C_{0}(X, \mathbb{R})$ at an eigenvalue $\lambda$ of $A_{2}$ on $\mathbb{R} \otimes_{\mathbb{Z}} \hat{Y}$.

We note that Walters avoids the use of the eigenfunction in his original statement. He prefers the equivalent condition that there exists no non-constant $\delta \in \hat{X}$ such that $\delta \circ A_{1}^{p}=\delta$ and $\delta \circ T_{1}^{p-1}(0)=\lambda^{p}$ for all such $p$.

Proof: Suppose that there exists a non-trivial equivariant map $g: \mathbb{R} \otimes_{\mathbb{Z}} \hat{Y} \rightarrow C_{0}(X, \mathbb{R})$. Then some element $y \in \mathbb{R} \otimes_{\mathbb{Z}} \hat{Y}$ satisfies $g(y) \neq 0$. Since $\hat{Y}$ is torsion, the module $M$ generated by $y$ is finite dimensional. By the Jordan decomposition theorem, $g\left(E_{\lambda}\right)$ is non-zero for some generalized eigenspace $E_{\lambda} \subset M$, which has a basis $v_{i}$ for $i=1, \ldots, k$ such that $A_{2}\left(v_{i}\right)=\lambda v_{i}+v_{i-1}$ (with $v_{0}=0$ ). If $i$ is the least index such that $g\left(v_{i}\right) \neq 0$ then $g\left(v_{i}\right)$ is an eigenfunction of $X$ at an eigenvalue of $A_{2}$. Hence if no such eigenfunction exists, then $\left(Y, T_{2}\right)$ is rigid.

$C(X, \mathbb{R})$ is an $S$-module even if $S$ acts on $X$ by arbitrary continuous transformations. So the algebraic condition of Theorem 2.8 applies to a general $S$-action on $X$, suggesting that a more general rigidity result is possible. According to the Gorin-Scheffer theorem, an affine transformation represents a homotopy class once we fix a base-point. So if all equivariant maps are affine, then this implies that no two equivariant maps are homotopic, once we fix a base-point. To fix our ideas, we consider the base-point preserving category with the unit element 0 as the base-point. Let $C(X, \mathbb{R})$ be the submodule of continuous maps such that $f(0)=0$. We say that $Y$ is homotopically rigid if no two base-point preserving equivariant maps into $Y$ are homotopic. 
Theorem 2.11: An S-algebraic dynamical system $Y$ is homotopically rigid with respect to a category of $S$-dynamical systems $X$ if and only if the only $\mathbb{Z}[S]$-module homomorphism between $\hat{Y}$ and $C_{p}(X, \mathbb{R})$ is the trivial homomorphism.

Proof: Suppose that $f$ and $g$ are two homotopic equivariant maps from $X$ to $Y$. Then $f-g$ is equivariant too and since it is homotopic to the trivial map, it can be lifted to an equivariant map $h: X \rightarrow L(Y)$. In the proof of Theorem 2.8 we found that such an equivariance is equivalent to a module homomorphism $\hat{Y} \rightarrow C(X, \mathbb{R})$ given by $\chi \mapsto(x \mapsto h(x)(\chi))$. Now we are dealing with pointed spaces and $f$ and $g$ preserve the base-point, so $h(0)(\chi)=0$ for all $\chi$. It follows that the module homomorphism has image in $C_{p}(X, \mathbb{R})$. Hence, the only module homomorphism between $\hat{Y}$ and $C_{p}(X, \mathbb{R})$ is the trivial one if and only if each homotopy class contains at most one equivariant map.

\section{Rigidity of hyperbolic automorphisms}

In the previous section we saw that many affine transformations are rigid in the sense that conjugating maps are affine. Conjugation is a restrictive notion for equivalence that is sometimes weakened to obtain other classifications. Conjugacy of flows, for instance, is far more restrictive than (orbit) topological equivalence of flows. Conjugation preserves some dynamical properties that topological equivalence does not. In this section we show that many algebraic dynamical systems remain rigid even if we replace conjugation by a relation that resembles topological equivalence for flows.

We consider groups as pointed spaces $(X, 0)$, and in what follows any map $f: X \rightarrow Y$ is assumed to be continuous and to preserve the base-point: $f(0)=0$. We only consider algebraic systems in this section for which $S=\mathbb{Z}$, and so we are dealing with an automorphism $A$ that acts on a compact connected metrisable abelian group. Recall that $W_{X}^{s}=\left\{x \in X: \lim _{n \rightarrow \infty} A^{n}(x)=0\right\}$ and $\widetilde{W}_{X}^{u}=\left\{x \in \underset{\sigma_{X}}{X}\right.$ : $\left.\lim _{n \rightarrow-\infty} A^{n}(x)=0\right\}$ denote the stable and unstable groups. Now let $\widetilde{W}_{X}^{s}$ and $\widetilde{W}_{X}^{u}$ be the stable and unstable manifolds of the lifted operator $L(A)$ on the tangent space $L(X)$. Notice that the image of $\widetilde{W}_{X}^{s}$ under the exponential map exp is equal to the path component of 0 in $W_{X}^{S}$ since the image of $L(X)$ is the path component of 0 .

Definition 3.1: We say that the algebraic dynamical system $(X, A)$ is hyperbolic if the tangent space decomposes as $L(X)=\widetilde{W}_{X}^{s} \oplus \widetilde{W}_{X}^{u}$.

For example, an expansive automorphism on a compact and connected group is hyperbolic. There are examples of non-expansive hyperbolic automorphisms, in particular there are examples on infinite-dimensional groups.

Note that a continuous base-point preserving map $f: X \rightarrow Y$ lifts to $L(f): L(X) \rightarrow L(Y)$. To see this, note that $f \circ \exp : L(X) \rightarrow Y$ is null-homotopic. Also note that the lift is unique by the connectedness of $L(X)$. If $X$ and $Y$ are hyperbolic, then we say that that $f$ respects the hyperbolic structure if $L(f)\left(\widetilde{W}_{X}^{s}\right) \subset \widetilde{W}_{Y}^{s}$ and $L(f)\left(\widetilde{W}_{X}^{u}\right) \subset \widetilde{W}_{Y}^{u}$.

Definition 3.2: We say that $(X, A)$ is hyperbolically rigid if any continuous map $f: X \rightarrow Y$ that respects the hyperbolic structure is a homomorphism. 
Lemma 3.3: Any $f: X \rightarrow Y$ that respects the hyperbolic structure is homotopic to a homomorphism that respects the hyperbolic structure.

Proof: According to the Gorin-Scheffer theorem there exists a unique homomorphism $h$ that is homotopic to $f$. Let $H_{t}$ be a homotopy between $f-h$ and the constant map. By Theorem 2.2 there exists a covering homotopy $\tilde{H}_{t}: X \rightarrow L(Y)$ of $H_{t}$. By the compactness of $X$, there is a uniform bound on the diameter of the arcs $\left\{\tilde{H}_{t}(x): 0 \leq t \leq 1\right\}$. Now $H_{t}$ lifts to a homotopy $L\left(H_{t}\right)$ between $L(f)$ and $L(h)$, and $L\left(H_{t}\right)$ is equal to $\tilde{H}_{t} \circ \exp$. So we find that $L(h)\left(\widetilde{W}_{X}^{s} \times\{0\}\right)$ is a linear subspace of $L(Y)$ that is within a bounded distance of the linear subspace $\widetilde{W}_{Y}^{S} \times\{0\}$ since the arcs $\left\{L\left(H_{t}\right)(x): 0 \leq t \leq 1\right\}$ are bounded. The linearity of these subspaces implies that the distance is zero, so the image of $L(h)$ is contained in $\widetilde{W}_{Y}^{s} \times\{0\}$. By the same argument $L(h)\left(\{0\} \times \widetilde{W}_{X}^{u}\right)$ is contained in $\{0\} \times \widetilde{W}_{Y}^{u}$.

Theorem 3.4: Let $(X, A)$ be a hyperbolic automorphism and let $W_{(X, 0)}^{s}$ and $W_{(X, 0)}^{u}$ be the path components of 0 in $W_{X}^{s}$ and $W_{X}^{u}$, respectively. If both $W_{(X, 0)}^{s}$ and $W_{(X, 0)}^{u}$ are dense in $X$, then $(X, A)$ is hyperbolically rigid.

Proof: Suppose that $f: X \rightarrow Y$ preserves the hyperbolic structure. By the previous lemma, $f$ is homotopic to a homomorphism $h$ with $h\left(W_{(X, 0)}^{S}\right) \subset W_{(Y, 0)}^{S}$ and $h\left(W_{(X, 0)}^{u}\right) \subset W_{(Y, 0)}^{u}$. Let $D:(X, 0) \rightarrow(L(Y), 0)$ be the lift of $h-f$. By connectivity, $D_{\tilde{W}^{u}}\left(W_{(X, 0)}^{s}\right) \subset \widetilde{W}_{Y}^{s} \times\{0\}$. By density, $D(X) \subset \widetilde{W}_{Y}^{s} \times\{0\}$. Similarly $D(X) \subset\{0\} \times \widetilde{W}_{Y}^{u}$. Hence $D(X)=0$ and $f$ is equal to $h$.

Expansive toral automorphisms, for example, satisfy the conditions of the theorem. More generally, if an expansive automorphism on a compact connected group has stable and unstable groups with non-trivial path components, then it is hyperbolically rigid. Thus, the natural extension of a hyperbolic toral endomorphism that has non-trivial stable and unstable groups provides another class of examples to which the theorem applies.

The shift on the dyadic solenoid, on the other hand, has a totally disconnected stable group. Even more so, it is not difficult to construct a homeomorphism $h$ on the dyadic solenoid that is not a homomorphism but that nevertheless preserves both the stable and the unstable group (so it preserves more than just the hyperbolic structure).

\section{Acknowledgement}

Part of this paper was written when the first author visited Delft University on NWO grant B 61-563.

\section{References}

[1] Arov, D.Z., 1963, Topological similitude of automorphisms and translations of compact commutative groups. Uspekhi Matematicheskikh Nauk, 18, 133-138.

[2] Bhattacharya, S., 2000, Orbit equivalence and topological conjugacy of affine actions on compact abelian groups. Monatshefte für Mathematik, 129, 89-96.

[3] Bhattacharya, S. and Ward, T., 2005, Finite entropy characterizes topological rigidity on connected groups. Ergodic Theory and Dynamical Systems, 25, 365-373. 
[4] Walters, P., 1969, Topological conjugacy of affine transformations of compact abelian groups. Transactions of the American Mathematical Society, 140, 95-107.

[5] Lin, V.Ja., 1970, Semi-invariant integration with values in a group, and some applications of it. Matematicheskii Sbornik (NS), 82, 233-259 [in Russian].

[6] Gorin, E.A., 1970, A function-algebra variant of the Bohr-van Kampen theorem. Matematicheskii Sbornik (NS), 82, 260-272 [in Russian].

[7] Scheffer, W., 1972, Maps between topological groups that are homotopic to homomorphisms. Proceedings of the American Mathematical Society, 33, 562-567. 